their size and shape are concerned, to the capacity of the strictured passage, and to the normal or abnormal course of the urethra in its entire length, most strictures can be overcome, and the urethra restored to its original condition, with as much security against relapse as by any other plan of treatment that has hitherto been devised, and with less risk of injury to the patient; and that the call for the knife is exceedingly exceptional.

Finsbury Place South, May 1861.

\section{DOES THE PERICARDIUM INVARIABLY BECOME ADHERENT TO THE HEART AFTER ACUTE PERICARDITIS AND RECOVERY?}

By F. Hughes Hewitr, M.D., Physician to the Worcester Dispensary.

The British Medical Journal, for March 16th, contains some remarks by Dr. Hinds, of Birmingham, on this very interesting question. The author thinks it should be answered in the negative. He admits the difficulty of "dealing with it very positively and conclusively," owing chiefly to the rarity of opportunities of examining the bodies of persons who have made recoveries from acute pericarditis. He assumes, correctly $I$ believe, that most pathologists bold with Dr. Watson, that the cessation of the to-and-fro sound is owing, not to absorption of the effused lymph, but to adhesion of the opposed surfaces of inflamed pericardium, and that " in this catalogue of apparent but unreal recoveries many of Bouillaud's cases of 'pericarditis terminating in health' ought to be included." He then relates two cases, occurring in his own practice, with the results of which he thinks "the principle above laid down is not consistent."

The first is the case of a gentleman, aged 27, who exhibited all the symptoms of pericarditis. He was treated by local bleeding, calomel and opium, and diuretics, and in eleven days the friction sound, together with " a little bruit with the first sound of the heart" had completely disappeared. This was three years ago, and the patient "shews not the slightest departure from robust and perfect health."

The second case is that of a child, aged about two years, who died after a few days illness, no heart symptoms having been detected during life. At the autopsy "the heart was found glued to the pericardium through. out its whole surface by a thick toughish layer of recent lymph."

Now, cases such as these are, comparatively speaking, common enough. Every practitioner who has seen much of acute disease must have met with instances of pericarditis in which all the symptoms, general and auscultatory, have disappeared, and apparent recovery has taken place; and others, which have terminated fatally, and in which obliteration of the pericardial cavity from recently effused lymph has been found after death. But what bearing they have upon the question with which Dr. Hinds heads his paper I am at a loss to understand. If his patient who had pericarditis three years ago were to die, and his pericardium were found to be free from adhesions, we should then have something like evidence before us. The grounds, however, upon which Dr. Hinds claims the cure as " complete and real", and arrives at the general conclusion that " pericarditis, with exudation of lymph on the surfaces, is a curable disease, and that in a strict and just sense of the term", appear to be :-

1. That he cannot reconcile the fact that his paitent, a young man, appears in perfect health three years after an attack of pericarditis, with the idea that adhesion took place at the time of the attack. "If the heart," he asks, " had been glued to the pericardium, could suc complete and lasting recovery have occurred? Surel not."

2. That he has never seen, and doubts the existenc of, cases of adherent pericardium, "if we except thos" from recent disease." "Do they ever occur?" he asks "Has any practitioner seen many, or any, such cases iQ which the patients, well of the cardiac, have died of some other disease, or even ' apparently' well of the. cardiac disease, living in apparent health for any cone siderable period afterwards?" "I have never myseifi met with any case of adherent pericardium, except frong recent disease."

I must confess that I read these remarks, especially the last sentence, with amazement. How long it has been established fact that old pericardial adhesion is occasionally met with at post mortem examinations, ustally quit: unexpectedly, I do not know. But many readers of this Journal must remember the discussion which toob place some twenty years ago between Drs. Barlow and Chevers as to whether such a pathological condition lead to hypertrophy, or to atrophy, of the muscular tissue of the heart. It has been thought by many physicians, by Dr. Munk among them, that it may be detected wit tolerable accuracy during life. Specimens of it are t be found in almost every museum. Dr. Wilks observes. (Pathological Anatomy, p. (65), "more frequently thafo this" (partial adhesion) "the heart is found universally adherent to its serous covering; thus, at the beginning of this year we found it three times in one week, ane quite unconnected with the disease of which the patien died, one indeed being an accident, and another a sur? gical case." Besides numerous instances in the Pathor logical Theatre at Guy's Hospital, I have met with tw or three in private practice; in one of these, a child aged 9 years, the pericardial adhesion, which was uni versal and evidently of long standing, was quite a minos feature, death having been caused by the suppuration of a mass of enlarged bronchial glands, which had opene $\Phi$ into the œsophagus, and penetrated the pulmonaro veins, giving rise to immense and rapidly fatal hæmor rhage.

Chronic adhesion of the pericardium, then, is a cons dition by no means unfrequently met with, and is com patible with the continuance for a very considerable pes riod, not merely of life, but of tolerable health, with a absence of cardiac symptoms, provided it be not como plicated with endocardial disease; in short, "simpte pericardial adhesion produces no appreciable untoward consequences." (Wilks, op. cit.)

As regards the second of the two propositions, alreadfs alluded to, with which Dr. Hinds sums up his paper viz., that pericarditis is a "curable" disease, I will on say that, without denying the possibility of the absorption. of the effused lymph, I think the evidence he brings forward wholly inadequate. The circumstance that slight and partial adhesions only are sometimes found seems to render it probable that a portion of the exudg tion, at all events, may be absorbed (supposing that the inflammation in these canos originally involved the whole of the membrane-which is not certain). Buf we ought, it seems to me, to suspend our judgment orr this point till we are in possession of well authenticatet cases of patients who have had unequivocal sympton $\overline{\widehat{S}}$ of pericarditis, who have recovered, have subsequent died, and have been found on inspection, free from pericardial adhesion.

Castor-orl Bread. The difficulty of taking castors oil, when but a mild effect of it is wanted on the bowele may be removed by kneading, etc., bread with it, insteag of butter or lard. Bread made with it will be foun quite as good to the taste, if made with fine castor-o $\$$ or finer than with butter, etc. (Med. Times.) 